JOURNAL OF INTEGRAL EQUATIONS

AND APPLICATIONS

Volume 6, Number 2, Spring 1994

\title{
CORRINGTON'S WALSH FUNCTION METHOD APPLIED TO A NONLINEAR INTEGRAL EQUATION
}

\author{
B.G. SLOSS AND W.F. BLYTH
}

\begin{abstract}
Corrington used Walsh functions to solve a particular nonlinear integral equation. This paper is concerned with the explicit formulation, development and extension of Corrington's Walsh function method to a class of nonlinear integral equations. In particular, given certain regularity conditions, error estimates are derived for the method in $L^{2}$ norm and sufficient conditions are given for the convergence of the method.
\end{abstract}

1. Introduction. Corrington [2] introduced a method for solving integral and differential equations using Walsh functions. This method is different from most classical iterative methods because higher order derivatives of the solution were obtained instead of the actual solution. Corrington applied this method to the solution of a nonlinear differential equation which has as its exact solution the Weierstrass elliptic function. However, little detail was given of how this method was to be implemented more generally.

In this paper we repeatedly use the Banach fixed point theorem in our study of a generalization of the integral equation which was solved by Corrington. We provide error estimates and sufficient conditions for convergence of this new method.

The nonlinear integral equation in $[\mathbf{2}]$ is

$$
u(x)=2\left(1+\int_{0}^{x}(x-t) u(t) d t\right)^{2}
$$

which arises from the initial value problem $d^{2} y / d x^{2}=2 y^{2}$ with $y(0)=1$ and $y^{\prime}(0)=0$ where $u$ is defined as $d^{2} y / d x^{2}$. The exact solution can be written in terms of the Weierstrass elliptic function as $y=$

Received by the editors on November 30, 1992, and in revised form on February 24, 1994

1991 Mathematics Subject Classification. Primary 45L10; Secondary 45G10, $42 \mathrm{C} 10$.

Copyright (C)1994 Rocky Mountain Mathematics Consortium 
$\mathcal{P}\left(x / \sqrt{3}+\omega_{2} / 4^{1 / 6} ; 0,4\right)$ (see $[\mathbf{1}]$ for notation, definitions and the value of the "half-period" $\left.\omega_{2}\right)$.

We consider the natural generalization of this integral equation:

$$
u(x)=\sum_{i=1}^{N} b_{i}\left(a_{i}(x)+\int_{0}^{x} k_{i}(x, t) u(t) d t\right)^{i}
$$

where $b_{i} \in \mathbf{R}, a_{i} \in L^{\infty}[0,1]$ and $k_{i} \in L^{\infty}\left[I^{2}\right], I^{2}=[0,1] \times[0,1]$. Consequently, $k_{i}$ and $a_{i}$ are essentially bounded on their respective intervals. We let

$$
K_{i}=\underset{(x, t) \in I^{2}}{\operatorname{ess} \sup _{i}}\left|k_{i}(x, t)\right|
$$

For some cases where the kernel functions are of convolution type, the solutions of equation (1) include the higher order derivatives of the elliptic functions and natural generalizations of these functions. The elliptic functions have found wide applications in many fields of science and engineering. Walsh function methods are attractive because their implementation usually involves the fast Walsh Fourier transform which is fast when compared with other finite transforms such as the trigonometric fast Fourier transform.

2. Notation. The $i^{\text {th }}$ Walsh function, ordered according to Paley $[\mathbf{5}, \mathbf{6}]$ is denoted by $W_{i} . P_{n}$ is the projection operator for projection onto the closure of the space spanned by the first $n$ Walsh functions: $P_{n}: L^{2}[0,1) \rightarrow \overline{\operatorname{span}}\left\{W_{i}: 0 \leq i \leq n-1\right\} . D_{n}(x)$ is the Dirichlet kernel,

$$
D_{n}(x)=\sum_{i=0}^{n-1} W_{i}(x)
$$

and $L_{n}$ is the Lebesgue constant for the Walsh system

$$
L_{n}=\int_{0}^{1}\left|D_{n}(t)\right| d t
$$

$\mathcal{I}: L^{2}[0,1) \rightarrow L^{2}[0,1)$ is the identity operator. 
Let

$$
\begin{aligned}
\omega(\delta) & =\omega(\delta, f) \\
& =\sup \left|f\left(x_{1}\right)-f\left(x_{2}\right)\right| \quad \text { for } x_{1}, x_{2} \in I, \quad\left|x_{2}-x_{1}\right| \leq \delta,
\end{aligned}
$$

where $I=[0,1]$ is the unit interval. The function $\omega(\delta)$ is called the modulus of continuity of $f$. If $\omega(\delta) \leq C^{\prime} \delta^{\alpha}$ for $\alpha \geq 0$, with $C^{\prime}$ independent of $\delta$, then $f \in \operatorname{Lip} \alpha$. A function $f$ belongs to Lip 1 if and only if it is the integral of a bounded function.

$S_{n}(x, f)$ is the $n$th partial sum of the Walsh Fourier series of $f$.

3. The Walsh functions. The Walsh functions were first introduced by Walsh [10]. However, we use the ordering due to Paley [5, 6]. Comprehensive accounts of the properties of Walsh functions and Walsh series can be found in either the book by B. Golubov et al. [3] or the book by F. Schipp et al. [8].

Every $f \in L^{k}[0,1), k \geq 1$, has associated with it a Walsh Fourier series

$$
f(x) \sim c_{0}+c_{1} W_{1}(x)+c_{2} W_{2}(x)+\cdots
$$

where the coefficients are given by

$$
c_{i}=\int_{0}^{1} f(x) W_{i}(x) d x .
$$

The collection of Walsh functions form an orthonormal complete collection of functions for the space $L^{2}[0,1)$ of square Lebesgue integrable functions; thus, every $f \in L^{2}[0,1)$ can be expanded as a series of Walsh functions with real coefficients. Then $f$ is the limit in $L^{2}$ of this series and the coefficients of the series coincide with $c_{n}$ above. Following Paley $[\mathbf{5}, \mathbf{6}]$ we may define the Walsh functions in terms of the Rademacher functions $\left\{r_{i}\right\}_{i=0}^{\infty}$. The Rademacher functions, published in $1922[\mathbf{7}]$, form an incomplete, with respect to $L^{2}[0,1)$, set of orthonormal functions. They are defined as follows.

$$
\begin{aligned}
r_{n} & : \mathbf{R} \rightarrow \mathbf{R}, \quad \forall n \in \mathbf{N} \cup\{0\} \\
r_{0}(x) & = \begin{cases}1 & \text { for } x \in[0,1 / 2), \\
-1 & \text { for } x \in[1 / 2,1),\end{cases} \\
r_{n}(x) & =r_{0}\left(2^{n} x\right), \quad \forall n \geq 1, \\
r_{n}(x+1) & =r_{n}(x) .
\end{aligned}
$$


The Walsh functions $\left\{W_{i}\right\}_{i=0}^{\infty}$ are defined as follows

$$
\begin{aligned}
W_{0}(x) & =1 \\
W_{n}(x) & =\prod_{i=1}^{r} r_{n_{i}}(x),
\end{aligned}
$$

where

$$
n=\sum_{i=1}^{r} 2^{n_{i}}, \quad n_{i+1}>n_{i}
$$

is the unique decomposition of $n$ into the sum of strictly increasing powers of 2 .

4. On the second order equation. The following theorem is a new result which indicates that, when $N=2$ and $b_{1}$ is zero, the integral equation (1) has a unique solution. This provides an analogous result to that obtained by Okrasinski [4] who considered the integral equation

$$
u^{\alpha}(x)=\int_{0}^{x} K(x-t) u(t) d t, \quad \alpha>1
$$

and some generalizations of this where the $u^{\alpha}$ is replaced by $W(u(x))$ with $W$ a convex function. However, the $\alpha=1 / 2$ was not treated and our second order equation can be regarded as a generalization of Okrasinski's $\alpha=1 / 2$ case.

Theorem 1. The integral equation

$$
z(x)=b\left(a(x)+\int_{0}^{x} k(x, t) z(t) d t\right)^{2},
$$

with $a \in L^{\infty}[0,1], b \in \mathbf{R}, k \in L^{\infty}\left[I^{2}\right]$, has a unique solution $u \in L^{2}$, where $u$ belongs to the closed ball $B_{c}=\left\{w \in L^{2}:\|w\|_{2} \leq c\right\}$ with

$$
0<c<\frac{1}{K}\left(\frac{1}{2 K|b|}-\|a\|_{\infty}\right)
$$

if

$$
\|a\|_{\infty}<\frac{1}{4 K|b|}
$$


Proof. The existence of a solution is guaranteed by the Corollary of Theorem 2 (established in the next section). Here we prove uniqueness. Suppose that $u$ and $u+v$ are solutions of (3) so

$$
\begin{aligned}
& v(x)=b\left\{2 a(x) \int_{0}^{x} k(x, t) v(t) d t\right. \\
&+2 \int_{0}^{x} k(x, t) u(t) d t \int_{0}^{x} k(x, t) v(t) d t \\
&\left.+\left(\int_{0}^{x} k(x, t) v(t) d t\right)^{2}\right\} .
\end{aligned}
$$

Let $\left\{\chi_{n}\right\}$ be a sequence of characteristic functions of the intervals $\left[0, A_{n}\right] \subset[0,1]$, and define $T_{n}: L^{2} \rightarrow L^{2}$ such that

$$
\begin{aligned}
T_{n} z(x)= & \chi_{n}(x) b\left\{2 a(x) \int_{0}^{x} k(x, t) z(t) d t\right. \\
& \left.+2 \int_{0}^{x} k(x, t) u(t) d t \int_{0}^{x} k(x, t) z(t) d t+\left(\int_{0}^{x} k(x, t) z(t) d t\right)^{2}\right\}
\end{aligned}
$$

For suitable $v_{1}, v_{2} \in L^{2}$, we have

$$
\begin{aligned}
T_{n} v_{1}(x)-T_{n} v_{2}(x)= & \chi_{n}(x) b\left\{2 a(x)+2 \int_{0}^{x} k(x, t) u(t) d t\right. \\
& \left.+\int_{0}^{x} k(x, t)\left(v_{1}(t)+v_{2}(t)\right) d t\right\} \\
& \cdot \int_{0}^{x} k(x, t)\left(v_{1}(t)-v_{2}(t)\right) d t \\
& \stackrel{\text { def }}{=} b z\left(u, v_{1}, v_{2}\right)(x) \int_{0}^{x} k(x, t)\left(v_{1}(t)-v_{2}(t)\right) d t
\end{aligned}
$$


By using Hölder's inequality and the monotone property,

$$
\begin{aligned}
& \int_{0}^{1}\left(T_{n} v_{1}-T_{n} v_{2}\right)^{2}(x) d x \\
& =\int_{0}^{1} b^{2} z^{2}\left(u, v_{1}, v_{2}\right)(x)\left(\int_{0}^{x} k(x, t)\left(v_{1}(t)-v_{2}(t)\right) d t\right)^{2} d x \\
& \leq b^{2} \operatorname{ess} \sup z^{2}\left(u, v_{1}, v_{2}\right)(x) \int_{0}^{1}\left(\int_{0}^{x}|k(x, t)|\left|v_{1}(t)-v_{2}(t)\right| d t\right)^{2} d x \\
& \leq b^{2} \operatorname{ess} \sup z^{2}\left(u, v_{1}, v_{2}\right)(x) \int_{0}^{1}\left(\int_{0}^{1}|k(x, t)|\left|v_{1}(t)-v_{2}(t)\right| d t\right)^{2} d x \\
& \leq b^{2} \operatorname{ess} \sup z^{2}\left(u, v_{1}, v_{2}\right)(x) \int_{0}^{1} \int_{0}^{1}|k(x, t)|^{2}\left|v_{1}(t)-v_{2}(t)\right|^{2} d t d x \\
& \leq b^{2} \operatorname{ess} \sup z^{2}\left(u, v_{1}, v_{2}\right)(x) K^{2} \int_{0}^{1}\left|v_{1}(t)-v_{2}(t)\right|^{2} d t .
\end{aligned}
$$

Letting $T_{n}: B_{c} \rightarrow L^{2}$ where $B_{c}=\left\{w \in L^{2}:\|w\|_{2} \leq c\right\}$,

$$
\begin{aligned}
\left|z\left(u, v_{1}, v_{2}\right)(x)\right| \leq & 2\|a\|_{\infty}+2 \int_{0}^{1}|k(x, t) \| u(t)| d t \\
& +\int_{0}^{1}\left|k(x, t) \| v_{1}(t)+v_{2}(t)\right| d t \quad \text { almost everywhere } \\
\leq & 2\|a\|_{\infty}+2\|k(x, \cdot)\|_{2}\|u\|_{2}+K\left\|v_{1}+v_{2}\right\|_{2} \\
\leq & 2\|a\|_{\infty}+2 K\left(\|u\|_{2}+c\right) .
\end{aligned}
$$

Combining these results, we have

$$
\left\|T_{n} v_{1}-T_{n} v_{2}\right\|_{2} \leq 2 K|b|\left(\|a\|_{\infty}+K\left(\|u\|_{2}+c\right)\right)\left\|v_{1}-v_{2}\right\|_{2} .
$$

Thus $T_{n}$ is contractive if the coefficient on the right hand side is less than one. This can be rewritten to give the condition

$$
c<\frac{1}{K}\left(\frac{1}{2 K|b|}-\|a\|_{\infty}\right)-\|u\|_{2} .
$$

Since $T_{n}$ maps the zero function to the zero function, the contractive condition (5) also gives $T_{n}: B_{c} \rightarrow B_{c}$. 
The derivation of this condition (5) assumed that $v_{1} \in B_{c}$. We now remove this restriction. Suppose that $v \neq 0$ is a solution of (4) which may lie outside the ball $B_{c}$. Then

$$
\begin{array}{r}
\left(\chi_{n} v\right)(x)=\chi_{n}(x) b\left\{2 a(x) \int_{0}^{x} k(x, t) v(t) d t\right. \\
+2 \int_{0}^{x} k(x, t) u(t) d t \int_{0}^{x} k(x, t) v(t) d t \\
\left.+\left(\int_{0}^{x} k(x, t) v(t) d t\right)^{2}\right\} \\
=b\left\{2 a(x) \chi_{n}(x) \int_{0}^{x} k(x, t) v(t) d t\right. \\
+2 \int_{0}^{x} k(x, t) u(t) d t \chi_{n}(x) \int_{0}^{x} k(x, t) v(t) d t \\
\left.\left.+\chi_{n}^{x}(x) \int_{0}^{x} k(x, t) v(t) d t\right)^{2}\right\} \\
+2 \int_{0}^{x} k(x, t) u(t) d t \chi_{n}(x) \int_{0}^{x} k(x, t) \chi_{n}(t) v(t) d t \\
\left.\quad+\left(\chi_{n}(x) \int_{0}^{x} k(x, t) \chi_{n}(t) v(t) d t\right)^{2}\right\} \\
=T_{n}\left(\chi_{n} v\right)(x), \quad k(x, t) \chi_{n}(t) v(t) d t
\end{array}
$$

showing that, for each $n, \chi_{n} v$ is a fixed point of $T_{n}$. Now since $\chi_{n} y \rightarrow 0$ in $L^{2}$, as $A_{n} \rightarrow 0$, for all $y \in L^{2}$ and $v \neq 0$ we may choose $\chi_{n} v \in B_{c}$ where $\chi_{n} v \neq 0$. But this is impossible because 0 is the unique solution in $B_{c}$. Thus the solution $u$ is unique if there exists some $c>0$ which satisfies condition (5). However, from Corollary 2 (of Theorem 2 in the next section), there exists a solution, $u$, such that

$$
\|u\|_{2}<\frac{1}{K}\left(\frac{1}{2 K|b|}-\|a\|_{\infty}\right)
$$

if

$$
\|a\|_{\infty}<\frac{1}{4 K|b|}
$$


Thus a suitable $c$ exists and the theorem is proven.

5. The general equation. The following theorem gives a sufficient condition for the general integral equation (1) to have a solution. Once again we use the classical theorem of Banach. The theorem supplies sufficient conditions for the existence of a solution to which the method of Corrington converges. Although the condition $K_{s}<1$ of Theorem 2 appears to be somewhat restrictive, by considering $A u+B$ where $u$ is a solution and $A, B$ are real constants, Theorem 2 may be used to prove the existence of a solution for a wide class of integral equations.

Theorem 2. There exists a solution to (1) in the closed ball $B_{c}$ provided that

$$
K_{s}^{2} \stackrel{\text { def }}{=} N_{b} \sum_{i=1}^{N} b_{i}^{2} i^{2}\left(\left\|a_{i}\right\|_{\infty}+K_{i} c\right)^{2 i-2} K_{i}^{2}<1
$$

and

$$
\sum_{i=1}^{N}\left|b_{i}\right|\left(|| a_{i} \|_{\infty}+K_{i} c\right)^{i}<c
$$

where $K_{i}=\operatorname{essup}_{(x, t) \in I^{2}}\left|k_{i}(x, t)\right|$ and $N_{b}$ is the number of nonzero $b_{i}$.

Proof. Let

$$
T^{1} v(x)=\sum_{i=1}^{N} b_{i}\left(a_{i}(x)+\int_{0}^{x} k_{i}(x, t) v(t) d t\right)^{i} .
$$

Now for $v_{i} \in B_{c}$, for suitable $c$,

$$
\begin{aligned}
T^{1} v_{2}(x)-T^{1} v_{1}(x)=\sum_{i=1}^{N} b_{i}\left\{\left(a_{i}(x)\right.\right. & \left.+\int_{0}^{x} k_{i}(x, t) v_{2}(t) d t\right)^{i} \\
& \left.-\left(a_{i}(x)+\int_{0}^{x} k_{i}(x, t) v_{1}(t) d t\right)^{i}\right\}
\end{aligned}
$$




$$
\begin{gathered}
=\sum_{i=1}^{N} b_{i}\left\{\left(a_{i}(x)+\int_{0}^{x} k_{i}(x, t) v_{2}(t) d t-a_{i}(x)\right.\right. \\
\left.-\int_{0}^{x} k_{i}(x, t) v_{1}(t) d t\right) \\
\cdot \sum_{j=0}^{i-1}\left(a_{i}(x)+\int_{0}^{x} k_{i}(x, t) v_{2}(t) d t\right)^{j} \\
\left.\cdot\left(a_{i}(x)+\int_{0}^{x} k_{i}(x, t) v_{1}(t) d t\right)^{i-1-j}\right\} \\
\stackrel{\text { def }}{=} \sum_{i=1}^{N} b_{i} \int_{0}^{x} k_{i}(x, t)\left(v_{2}(t)-v_{1}(t)\right) d t F_{i}\left(x, v_{1}, v_{2}\right) .
\end{gathered}
$$

So

$$
\begin{aligned}
\left\|T^{1} v_{1}-T^{1} v_{2}\right\|_{2}^{2} \leq N_{b} \sum_{i=1}^{N} b_{i}^{2} \int_{0}^{1}\left(\int_{0}^{x}\left|k_{i}(x, t)\left(v_{2}(t)-v_{1}(t)\right)\right| d t\right)^{2}\left(x, v_{1}, v_{2}\right) d x \\
\leq N_{b} \sum_{i=1}^{N} b_{i}^{2} \operatorname{ess} \sup F_{i}^{2}\left(x, v_{1}, v_{2}\right) \\
\leq N_{b} \sum_{i=1}^{N} b_{i}^{2} \operatorname{ess} \sup F_{i}^{2}\left(x, v_{1}, v_{2}\right) \\
\left.\quad \int_{0}^{x}\left|k_{i}(x, t)\right|\left|v_{2}(t)-v_{1}(t)\right| d t\right)^{2} d x \\
\leq N_{b} \sum_{i=1}^{N} b_{i}^{2} \operatorname{ess} \sup F_{i}^{2}\left(x, v_{1}, v_{2}\right) K_{i}^{2}
\end{aligned}
$$




$$
\begin{gathered}
\cdot\left(\int_{0}^{1}\left|v_{2}(t)-v_{1}(t)\right| d t\right)^{2} \\
\leq N_{b} \sum_{i=1}^{N} \operatorname{ess} \sup F_{i}^{2}\left(x, v_{1}, v_{2}\right) K_{i}^{2} \int_{0}^{1}\left|v_{2}(t)-v_{1}(t)\right|^{2} d t,
\end{gathered}
$$

by Hölder's inequality. But

$$
\begin{aligned}
\left|F_{i}\left(x, v_{1}, v_{2}\right)\right| \leq & \sum_{j=0}^{i-1}\left(\left|a_{i}(x)\right|+\int_{0}^{x}\left|k_{i}(x, t) v_{2}(t)\right| d t\right)^{j} \\
& \cdot\left(\left|a_{i}(x)\right|+\int_{0}^{x} \mid k_{i}(x, t) v_{1}(t) d t\right)^{i-1-j} \\
\leq & \sum_{j=0}^{i-1}\left(\left\|a_{i}\right\|_{\infty}+K_{i} \int_{0}^{x}\left|v_{2}(t)\right| d t\right)^{j} \\
& \cdot\left(\left\|a_{i}\right\|_{\infty}+K_{i} \int_{0}^{x}\left|v_{1}(t)\right| d t\right)^{i-1-j} \\
\leq & \sum_{j=0}^{i-1}\left(\left\|a_{i}\right\|_{\infty}+K_{i} \int_{0}^{1}\left|v_{2}(t)\right| d t\right)^{j} \\
& \cdot\left(\left\|a_{i}\right\|_{\infty}+K_{i} \int_{0}^{1}\left|v_{1}(t)\right| d t\right)^{i-1-j} \\
\leq & \sum_{j=0}^{i-1}\left(\left\|a_{i}\right\|_{\infty}+K_{i}\left\|v_{2}\right\|_{2}\right)^{j}\left(\left\|a_{i}\right\|_{\infty}+K_{i}\left\|v_{1}\right\|_{2}\right)^{i-1-j},
\end{aligned}
$$

by Hölder's inequality,

$$
\begin{aligned}
& \leq \sum_{j=0}^{i-1}\left(\left\|a_{i}\right\|_{\infty}+K_{i} c\right)^{j}\left(\left\|a_{i}\right\|_{\infty}+K_{i} c\right)^{i-1-j} \\
& \leq i\left(\left\|a_{i}\right\|_{\infty}+K_{i} c\right)^{i-1}
\end{aligned}
$$

giving

$$
\operatorname{ess} \sup \left|F_{i}\left(x, v_{1}, v_{2}\right)\right| \leq i\left(\left\|a_{i}\right\|_{\infty}+K_{i} c\right)^{i-1} .
$$

Consequently $T^{1}$ is a contraction mapping if

$$
K_{s}^{2} \stackrel{\text { def }}{=} N_{b} \sum_{i=1}^{N} b_{i}^{2} i^{2}\left(\left\|a_{i}\right\|_{\infty}+K_{i} c\right)^{2 i-2} K_{i}^{2}<1 .
$$


To establish that $T^{1}: B_{c} \rightarrow B_{c}$, consider

$$
\begin{aligned}
\|\left(a_{i}(x)+\int_{0}^{x} k_{i}(x, t) v\right. & (t) d t)^{i} \|_{2}^{2} \\
& =\int_{0}^{1}\left(a_{i}(x)+\int_{0}^{x} k_{i}(x, t) v(t) d t\right)^{2 i} d x \\
& \leq \int_{0}^{1}\left(\left|a_{i}(x)\right|+\int_{0}^{x}\left|k_{i}(x, t)\right||v(t)| d t\right)^{2 i} d x \\
& \leq \int_{0}^{1}\left(\left|a_{i}(x)\right|+\int_{0}^{1}\left|k_{i}(x, t)\right||v(t)| d t\right)^{2 i} d x \\
& \leq \int_{0}^{1}\left(\left\|a_{i}\right\|_{\infty}+K_{i} \int_{0}^{1}|v(t)| d t\right)^{2 i} d x \\
& =\left(\left\|a_{i}\right\|_{\infty}+K_{i} \int_{0}^{1}|v(t)| d t\right)^{2 i} \\
& \leq\left(\left\|a_{i}\right\|_{\infty}+K_{i} c\right)^{2 i} .
\end{aligned}
$$

Thus

$$
\left\|T^{1} v\right\|_{2} \leq \sum_{i=1}^{N}\left|b_{i}\right|\left(\left\|a_{i}\right\|_{\infty}+K_{i} c\right)^{i}
$$

giving $T^{1}: B_{c} \rightarrow B_{c}$ if

$$
\sum_{i=1}^{N}\left|b_{i}\right|\left(|| a_{i} \|_{\infty}+K_{i} c\right)^{i}<c .
$$

Theorem 2 follows by Banach's theorem.

Remark. The conditions for Theorem 2 can be simplified by introducing stronger regularity conditions. For example, there exists a solution to (1) in the unit ball if

$$
\left|b_{i}\right| \leq \frac{1}{N_{b} i} \quad \text { and } \quad\left\|a_{i}\right\|_{\infty}+K_{i}<1
$$

for each $i$. 
Corollary. There exists a solution $u$ to the second order equation

$$
u(x)=b\left(a(x)+\int_{0}^{x} k(x, t) u(t) d t\right)^{2}
$$

where $u$ belongs to the closed ball $B_{c}=\left\{w \in L^{2}:\|w\|_{2} \leq c\right\}$ with

$$
0<c<\frac{1}{K}\left(\frac{1}{2 K|b|}-\|a\|_{\infty}\right)
$$

if

$$
\|a\|_{\infty}<\frac{1}{4 K|b|}
$$

Proof. Theorem 2 gives the following sufficient conditions for the existence of a solution

$$
|b|\left(|| a \|_{\infty}+K c\right)^{2}<c
$$

and

$$
2|b|\left(\|a\|_{\infty}+K c\right) K<c .
$$

The first of these inequalities is equivalent to $c<\bar{c}$ where

$$
\bar{c}=\frac{1}{K}\left(\frac{1}{2 K|b|}-\|a\|_{\infty}\right) .
$$

The second inequality can be rewritten as

$$
|b| K^{2} c^{2}+\left(2 K|b|\|a\|_{\infty}-1\right) c+|b|\|a\|_{\infty}^{2}<0
$$

which is satisfied by $c \in\left(c_{-}, c_{+}\right)$, where

$$
c_{ \pm}=\frac{1-2 K|b||| a \|_{\infty} \pm \sqrt{1-4 K|b|\|a\|_{\infty}}}{2|b| K^{2}} .
$$

The regularity condition

$$
4 K|b||| a \|_{\infty}<1
$$


is required to ensure that $c_{-}$and $c_{+}$are real (and positive). Note that $\bar{c}=\left(c_{-}+c_{+}\right) / 2$ so that, if the regularity condition is satisfied, any $c \in\left(c_{-}, \bar{c}\right)$ satisfies both of the inequalities from Theorem 2 and the Corollary is established.

The existence of a solution of (1) is guaranteed by Theorem 2. In the previous section, Theorem 1 also provided uniqueness for the second order case. However, more generally, we would expect that solutions of (1) would exhibit bifurcation behavior. The following theorem describes this for the case where our class of nonlinear equations is restricted to have all $a_{i}$ zero.

Theorem 3. For

$$
u(x)=b \sum_{i=1}^{N}\left(\int_{0}^{x} k_{i}(x, t) u(t) d t\right)^{i}
$$

with $b \in \mathbf{R}, u \in C[0,1]$ and the linearized problem

$$
u(x)=b_{0} \int_{0}^{x} k_{1}(x, t) u(t) d t
$$

with corresponding adjoint equation

$$
u^{*}(x)=b_{0} \int_{0}^{x} k_{1}(t, x) u^{*}(t) d t
$$

if $b_{0}$ is a simple characteristic number of $(7)$, then $(b, u)=\left(b_{0}, 0\right)$ is a bifurcation point of (6) when the following are satisfied.

1. Every $k_{i}$ is continuous on $[0,1] \times[0,1]$.

2. If $u_{1}$ is an eigensolution of (7) and $u_{1}^{*}$ is an eigensolution of (8), then $\left(u_{1} \mid u_{1}^{*}\right) \neq 0$ where $(x \mid y)=\int_{0}^{1} x(t) y(t) d t$. In particular, this is satisfied if $k_{1}(x, t) \geq 0$.

Proof. Setting

$$
N u=b \sum_{i=2}^{N}\left(\int_{0}^{x} k_{i}(x, t) u(t) d t\right)^{i}
$$


gives the Gateau derivative

$$
N_{u} h=b \sum_{i=2}^{N} i\left(\int_{0}^{x} k_{i}(x, t) u(t) d t\right)^{i-1} \int_{0}^{x} k_{i}(x, t) h(t) d t
$$

with $N_{u}(0)=0$. The proof follows that of example 8.30 of Zeidler [11].

口

6. Error estimates for Corrington's method. We interpret the method of Corrington as consisting of creating solutions $u^{n}$ by using iterates $u_{m}^{n}$ where

$$
\begin{aligned}
u_{m+1}^{n}(x) & =P_{n}\left(\sum_{i=1}^{N} b_{i}\left(a_{i}(x)+\int_{0}^{1} k(x, t) u_{m}^{n}(t) d t\right)^{i}\right) \\
& =T^{n}\left(u_{m}^{n}\right) .
\end{aligned}
$$

We show that there exist $u^{n} \in L^{2}[0,1)$ such that

$$
u_{m}^{n} \stackrel{L_{2}}{\rightarrow} u^{n} \stackrel{L^{2}}{\rightarrow} u .
$$

In the following we give error estimates for Corrington's method. In a previous paper $[\mathbf{9}]$, it was shown that the Corrington method for the linear case converges with order 2. That is, the error in the Walsh coefficients decreases by a factor of four as the number of Walsh terms is doubled. Similarly for the nonlinear case here, we show that, subject to sufficient regularity of the $k_{i}$ and $a_{i}$, the method converges quadratically.

Theorem 4. If $a_{i} \in \operatorname{Lip} \alpha$ and $k_{i}(\cdot, t) \in \operatorname{Lip} \alpha$ for all $t$, then we have the error estimates for Corrington's method:

$$
\left\|u_{m}^{n}-u\right\|_{2} \leq \frac{K_{s}^{m}}{1-K_{s}}\left\|u_{1}^{n}-u_{2}^{n}\right\|_{2}+\sum_{i=1}^{N}\left|b_{i}\right| D_{n, i}
$$

where $K_{s}^{2}=N_{b} \sum_{i=1}^{N} b_{i}^{2} i^{2}\left(\left\|a_{i}\right\|_{\infty}+K_{i} c\right)^{2 i-2} K_{i}^{2}, c$ is the radius of the ball $B_{c}$, and

$$
D_{n, i}= \begin{cases}O\left(n^{-\alpha} \log n\right) & \text { for arbitrary } n \\ O\left(n^{-\alpha}\right) & \text { for } n=2^{N} .\end{cases}
$$


Proof. Now

$$
\begin{aligned}
\left\|u_{m}^{n}-u\right\|_{2} & =\left\|u_{m}^{n}-u^{n}+u^{n}-u\right\|_{2} \\
& \leq\left\|u_{m}^{n}-u^{n}\right\|_{2}+\left\|u^{n}-u\right\|_{2} \\
& \leq \frac{K_{s}^{m}}{1-K_{s}}\left\|u_{1}^{n}-u_{2}^{n}\right\|_{2}+\left\|u^{n}-u\right\|_{2} .
\end{aligned}
$$

Let

$$
\begin{aligned}
\left\|u^{n}-u\right\|_{2}= & \| \sum_{i=1}^{N} b_{i} P_{n}\left(a_{i}(x)+\int_{0}^{x} k_{i}(x, t) u(t) d t\right)^{i} \\
& -\sum_{i=1}^{N} b_{i}\left(a_{i}(x)+\int_{0}^{x} k_{i}(x, t) u(t) d t\right)^{i} \|_{2} \\
\leq & \sum_{i=1}^{N}\left|b_{i}\right|\left\|\left(P_{n}-I\right)\left(a_{i}(x)+\int_{0}^{x} k_{i}(x, t) u(t) d t\right)^{i}\right\|_{2} \\
\stackrel{\text { def }}{=} & \sum_{i=1}^{N}\left|b_{i}\right|\left\|\left(P_{n}-I\right) H_{i}(x)\right\|_{2} .
\end{aligned}
$$

We now establish that $H_{i}$ belongs to $\operatorname{Lip} \alpha$ :

$$
\begin{aligned}
H_{i}(x+h)-H_{i}(x)= & \left(a_{i}(x+h)+\int_{0}^{x+h} k_{i}(x+h, t) u(t) d t\right)^{i} \\
& -\left(a_{i}(x)+\int_{0}^{x} k_{i}(x, t) u(t) d t\right)^{i}
\end{aligned}
$$

which, on factorization, gives

$$
\begin{array}{r}
H_{i}(x+h)-H_{i}(x)=\left\{a_{i}(x+h)-a_{i}(x)+\int_{0}^{x+h} k_{i}(x+h, t) u(t) d t\right. \\
\left.-\int_{0}^{x} k_{i}(x, t) u(t) d t\right\} G_{i}(x, h)
\end{array}
$$


where clearly $G_{i}(x, h) \rightarrow G_{i}(x)$, generally nonzero, as $h \rightarrow 0$. So, for $a_{i}$ and $k(\cdot, t)$ belonging to $\operatorname{Lip} \alpha$ for all $t$,

$$
\begin{aligned}
H_{i}(x+h)-H_{i}(x)= & \left\{a_{i}(x+h)-a_{i}(x)+\int_{0}^{x}\left(k_{i}(x+h, t)-k(x, t)\right) u(t) d t\right. \\
& \left.\quad+\int_{x}^{x+h} k_{i}(x+h, t) u(t) d t\right\} G_{i}(x, h) \\
= & O\left(h^{\alpha}\right)+O\left(h^{\alpha}\right)+O(h) \\
= & O\left(h^{\alpha}\right) \quad \text { as } h \rightarrow 0 .
\end{aligned}
$$

So

$$
\sup _{h \leq 1}|H(x+h)-H(x)|=O\left(h^{\alpha}\right)
$$

which implies that $H \in \operatorname{Lip} \alpha$.

Therefore, from [3], with $n=2^{J}+j$ for $j \leq 2^{J}$,

$$
\begin{aligned}
\left|\left(\mathcal{I}-P_{n}\right) H_{i}(x)\right| & =\left|S_{n}\left(x, H_{i}\right)-H_{i}(x)\right| \\
& \leq \omega\left(\frac{1}{2^{J}}, H_{i}\right)\left(2+\frac{1}{2} L_{n}\right) \\
& = \begin{cases}O\left(n^{-\alpha} \log n\right) & \text { for arbitrary } n \\
O\left(n^{-\alpha}\right) & \text { for } n=2^{N} .\end{cases} \\
& =D_{n, i}\left(H_{i}\right) .
\end{aligned}
$$

Thus, we have our result:

$$
\left\|u_{m}^{n}-u\right\|_{2} \leq \frac{K_{s}^{m}}{1-K_{s}}\left\|u_{1}^{n}-u_{2}^{n}\right\|_{2}+\sum_{i=1}^{N}\left|b_{i}\right| D_{n, i}\left(H_{i}\right) .
$$

It is natural to use the $n=2^{J}$ series expansion when using Walsh function methods, in which case

$$
\left\|u_{m}^{n}-u\right\|_{2} \leq D / n^{\alpha}+f_{m}
$$

where $n=2^{J}, f_{m} \rightarrow 0$ as $m \rightarrow \infty$ and $D$ is independent of $n$. Usually we have the regularity condition $\alpha=1$ and so

$$
\left\|u_{m}^{2^{J+1}}-u\right\|_{2} \sim \frac{1}{2}\left\|u_{m}^{2^{J}}-u\right\|_{2}
$$


Thus the error in global $L^{2}$ norm on $[0,1)$ is of first order. But the Walsh series of order $2^{J}$ is constant on intervals of measure $2^{-J}$. Over these intervals the local error is of order 2. This implies that the error in the coefficients of the Walsh series is of order 2. It is interesting to note that this is the same result as was obtained in [9] for the linear case of the Corrington method.

7. Conclusion. The method due to Corrington has been extended to a class of nonlinear integral equations which include the important elliptic equations. Sufficient conditions for convergence and error estimates have been derived. The error estimates developed here indicate that, subject to regularity conditions, the method is second order accurate when series with $2^{J}$ terms are used.

We have also obtained several new results concerning (i) the existence of solutions of our general nonlinear integral equation, (ii) location of multiple solutions where our class of equations is restricted to have all $a_{i}$ zero and (iii) uniqueness of the solution of the second order nonlinear equation.

\section{REFERENCES}

1. M. Abramowitz and I.A. Stegun, Handbook of mathematical functions, Dover, New York, 1965.

2. M.S. Corrington, A solution of differential and integral equations with Walsh functions, IEEE Trans. Circuit Theory, CT-20, 5 (1973), 470-476.

3. B.E. Golubov, A. Efimov and V. Skvortsov, Walsh series and transformations, Kluwer, Netherlands, 1991.

4. W. Okrasinski, Non-negative solutions of some nonlinear integral equations, Ann. Polon. Math. 44 (1984), 209-218.

5. R.E.A.C. Paley, A remarkable series of orthogonal functions (I), London Math. Soc. 34 (1932), 241-264.

6. - A remarkable series of orthogonal functions (II), London Math. Soc. 34 (1932), 265-279.

7. H. Rademacher, Einige sätze über Reihen von Allgemein Orthogonalen funktionen, Math. Annal. 87 (1922), 112-138.

8. F. Schipp, W.R. Wade, P. Simon and J. Pal, Walsh series: An introduction to dyadic harmonic analysis, Adam Hilger, New York, 1990.

9. B.G. Sloss and W.F. Blyth, A-priori error estimates for Corrington's Walsh function method, RMIT Mathematics Dept. Research Report 17 (1991). 
10. J.L. Walsh, A closed set of normal orthogonal functions, Amer. J. Math. 45 (1923), 5-24.

11. E. Zeidler, Nonlinear functional analysis and its applications, Vol. 1, Springer-Verlag, New York, 1986.

Department of Mathematics, Royal Melbourne Institute of Technology, Melbourne, Australia 3001 